\title{
The effects of traditional Chinese medicine sensory stimulation combined with transcranial direct current stimulation on deglutition and related complications in stroke patients with dysphagia: a randomized trial
}

\author{
Ying Lu ${ }^{1}$, Wangyang Zhou ${ }^{1}$, Yachen $\operatorname{Lin}^{2}$, Yupeng $\mathrm{Du}^{1}$, Xiaojing Zhang ${ }^{3}$ \\ ${ }^{1}$ Department of Cardiopulmonary Rehabilitation, The Third Affiliated Hospital of Zhejiang Chinese Medical University, Hangzhou, China; \\ ${ }^{2}$ Department of Cardiopulmonary Rehabilitation, Zhejiang Rehabilitation Medical Center, Hangzhou, China; ${ }^{3}$ Department of Nursing, The Third \\ Affiliated Hospital of Zhejiang Chinese Medical University, Hangzhou, China \\ Contributions: (I) Conception and design: Y Lu, W Zhou, X Zhang; (II) Administrative support: Y Lin, Y Du; (III) Provision of study materials \\ or patients: Y Lu, Y Lin, X Zhang; (IV) Collection and assembly of data: All authors; (V) Data analysis and interpretation: Y Lu, X Zhang; (VI) \\ Manuscript writing: All authors; (VII) Final approval of manuscript: All authors. \\ Correspondence to: Xiaojing Zhang. Department of Nursing, The Third Affiliated Hospital of Zhejiang Chinese Medical University, No. 219, \\ Moganshan Road, Hangzhou 310005, China. Email: ly18058700796@163.com.
}

\begin{abstract}
Background Stroke is a serious manifestation of a variety of cerebrovascular diseases and can cause significant morbidity and mortality. This study explored the effects of traditional Chinese medicine (TCM) sensory stimulation combined with transcranial direct current stimulation (tDCS) on swallowing function and related complications in stroke patients with dysphagia.
\end{abstract}

Methods: A total of 60 stroke patients with deglutition dysfunction admitted to our hospital from August 2019 to January 2021 were selected as research subjects. The patients were randomized into 3 different treatment groups, namely, the TCM group, the tDCS group, and the TCM combined with tDCS group (the combined group). Water swallowing tests (WSTs) were conducted to compare the swallowing function before and after treatment. Video fluoroscopic swallowing studies (VFSS) were conducted to analyze the scores of the oral phase, pharyngeal phase, and degree of aspiration. Brain activation and brain volume were examined using functional magnetic resonance imaging (fMRI).

Results: The WST and VFSS scores in the combined treatment group were significantly higher than that observed in the other two groups $(\mathrm{P}<0.05)$. The $\mathrm{fMRI}$ demonstrated that the brain activation volume in the combined treatment group was also greater than that in the other two groups $(\mathrm{P}<0.05)$.

Conclusions: TCM combined with tDCS significantly improved deglutition and complications in stroke patients.

Trial Registration: Chinese Clinical Trial Registry ChiCTR-TRC-12001972.

Keywords: Traditional Chinese medicine sensory stimulation (TCM sensory stimulation); Jieyudan powder stick stimulation; transcranial direct current stimulation (tDCS); dysphagia; water swallowing test (WST)

Submitted Apr 08, 2021. Accepted for publication Jun 01, 2021.

doi: 10.21037/apm-21-1055

View this article at: http://dx.doi.org/10.21037/apm-21-1055 


\section{Introduction}

Worldwide, there is an increasing incidence of stroke which is a leading cause of disability, morbidity, and mortality. It is positively correlated with the rise in the aging population, the fast pace of life, and poor health awareness (1). The pathogenesis of strokes includes various factors such as damage to the blood vessels or tissues in the brain, which eventually leads to acute cerebral blood circulation disorders. The complications of stroke include impairments in motor function and breathing, cognitive impairment, aphasia, and disturbances of consciousness, sensation, and perception (2). The most common symptom of stroke is dysphagia, accounting for about $30-65 \%$ of all complications with the ingested food failing to reach the stomach. If patients are not diagnosed or treated in a timely manner, dysphagia can lead to aspiration pneumonia, malnutrition, reduced resistance, dehydration, asphyxia, and recurrent pulmonary infections, all of which can seriously affect the morbidity and mortality patients (3).

According to traditional Chinese medicine (TCM) theory, the underlying pathogenesis of stroke is a dysfunction of the yin and yang, and a disordered flow of qi and blood. Stroke is inextricably linked with wind, fire, sputum, and stagnation (4). Dysphagia is caused by disordered internal wind movement, phlegm and blood stasis, and blockage of qi and blood. Many studies have demonstrated that TCM plays an important role in improving deglutition after a stroke (5).

Transcranial direct current stimulation (tDCS) is a non-invasive brain-stimulation technique which acts by directly applying weak current signals on the surface of the cerebral cortex. Specifically, tDCS plays a pivotal role in regulating the excitability of the cerebral cortex, as well as the activation and remodeling of the central cortex (6). Compared with traditional brain stimulation strategies such as transcranial magnetic stimulation (TMS) and deep brain stimulation, tDCS has been shown to significantly decrease the severity and incidence of headaches, dizziness, nausea, and insomnia (7).

A total of 60 stroke patients with deglutition dysfunction who were admitted to our hospital from August 2019 to January 2021 were enrolled in this study. This report examined the effects of TCM sensory stimulation and tDCS on deglutition and related complications in stroke patients with dysphagia.

We present the following article in accordance with the CONSORT reporting checklist (available at http://dx.doi. org/10.21037/apm-21-1055).

\section{Methods}

\section{General information}

A total of 60 stroke patients with deglutition dysfunction who were admitted to the Third Affiliated Hospital of Zhejiang Chinese Medical University from August 2019 to January 2021 were selected for this study. The following inclusion criteria were applied: (I) patients with confirmed cerebral hemorrhage or cerebral infarction by computed tomography (CT) or magnetic resonance imaging (MRI); (II) patients diagnosed with dysphagia following the water swallowing test (WST); (III) patients aged between 60 to 80 years; (IV) patients who satisfied the TCM syndromes of wind phlegm and blood stasis; and (V) patients with stable vital signs and who fully understood the study and voluntarily provided informed consent. Patients were excluded from the study if they presented with the following: (I) dysphagia not caused by stroke; (II) presence of metal in the body, such as a cardiac pacemaker; (III) acute illness or bleeding diathesis; (IV) blood pressure greater than $180 \mathrm{mmHg}$ systolic and/or $120 \mathrm{mmHg}$ diastolic; (V) cardiac failure, pulmonary failure, or renal failure; (VI) other critical conditions; and (VII) conscious or cognitive impairment. Patients were also excluded if they did not satisfy the TCM syndromes of wind phlegm and blood stasis. Randomization was completed using a computer-generated numbers table from the statistics package SPSS18.0. Enrolled patients were further randomly allocated into three groups, namely, the TCM group, the tDCS group, and the TCM combined with tDCS group (the combined treatment group). This study used a single-blinded method involving different personnel for experimentation, observation, data collection, and statistical analyses. All procedures performed in this study involving human participants were in accordance with the Declaration of Helsinki (as revised in 2013). The study was approved by the Research Ethics Committee of the Third Affiliated Hospital of Zhejiang Chinese Medical University (No.: ZSLL-JS-2020-002-01).

\section{Treatments}

All patients with cerebral hemorrhage or cerebral infarction were given conventional treatment in the neurology department to control and prevent complications. Symptomatic therapy and supporting treatments were carried 
out according to the condition of the patients. Different levels of intervention were administered as required.

\section{The TCM group}

The TCM regimen was Jieyudan powder stick stimulation, consisting of Bai aconite $10 \mathrm{~g}$, Shicalamus $10 \mathrm{~g}$, Yuanzhizhi $10 \mathrm{~g}$, Tianma $9 \mathrm{~g}$, Quan Scorpio $3 \mathrm{~g}$, Qianghuo $10 \mathrm{~g}$, Nanxing $10 \mathrm{~g}$, Muxiang $10 \mathrm{~g}$, and licorice $6 \mathrm{~g}$. One dose of Jieyudan was mixed with $500 \mathrm{~mL}$ of water and decocted to $200 \mathrm{~mL}$. After removing the dregs, the liquid was poured into ice trays of $4 \mathrm{~cm} \times 10 \mathrm{~cm} \times 3 \mathrm{~cm}$, and a cotton stick was inserted into each ice tray. Finally, the medicine popsicles were packed in a plastic bag and frozen for further use. The medical staff administered the Jieyudan powder sticks by swabbing the patient's palatoglossal arch, soft palate, palatopharyngeal arch, pharynx wall, both sides of the tongue body, and the tongue surface. The movements should be gentle and slow. During the stimulation intervals, patients were asked to make continuous "ee-mmm" sounds and try to maneuver their cheeks to show their teeth. They were also encouraged to perform the saliva swallowing test. Patients were treated twice daily, 30 minutes per treatment, for 30 consecutive days.

\section{The tDCS group}

The Transcranial Direct Current Stimulation machine (IS200 Intelligent Electrical Stimulator; Sichuan Intelligent Electronic Industry Company, Sichuan, China) was placed in a quiet room. A $5 \mathrm{~cm} \times 5 \mathrm{~cm}$ pole piece was selected and the current intensity was adjusted to $2 \mathrm{~mA}$. To increase the full electrode-skin contact, sponge pads were soaked in saline before use. The positive pole was placed on the left dorsal lateral prefrontal cortex (the stimulation area $8 \mathrm{~cm}$ before the central $\mathrm{CZ}$ point and $6 \mathrm{~cm}$ before the parietal opening were selected using the $\mathrm{F} 3$ and $\mathrm{F} 4$ regions of the international electroencephalogram system standard). The negative pole was placed on the right orbitofrontal region. Patients were treated once daily, 20 minutes each time, for 30 consecutive days.

\section{The TCM combined with tDCS group}

Patients in this group were treated as for the TCM group and the tDCS group.

\section{Evaluation markers}

Water swallowing test

The WST proposed by Toshio Wada in Japan in 1982 was performed to evaluate the swallow function before and after 30 consecutive days of treatment. Patients swallowed $30 \mathrm{~mL}$ of warm water and were monitored for the presence of a choking cough. The WST was divided into grades. Grade I represented patients who successfully swallowed $30 \mathrm{~mL}$ of warm water in one attempt within 5 seconds and they were given 0 points. Grade II referred to patients who swallowed in 2 or more gulps within 5-10 seconds without choking, and they were allocated 2 points. Grade III referred to patients who could swallow in 1 attempt within 5-10 seconds, but with a cough during swallowing, and they were given 4 points. Grade IV referred to patients who swallowed in 2 or more gulps within 5-10 seconds with choking during swallowing, and they were given 6 points. Grade $\mathrm{V}$ referred to patients who coughed several times and experienced difficulty swallowing the full volume within 10 seconds, and they were allocated 8 points. Thus, the score is inversely proportional to the degree of dysphagia, that is, higher scores indicated poorer swallowing function. A treatment was considered to have a curative effect, obvious effect, be effective, or ineffective depending on changes in the WST grade post-treatment. A patient with WST grade I was considered to be cured. If the grade of WST was increased by 2 grades or more, the treatment was considered to have an obvious effect in the patient. If the grade of WST increased by 1 grade or more, it was deemed to be effective. If the treatment did not change the grading of WST, it was deemed ineffective. The total effective rate $=$ (number of cured cases + number of obvious effect cases + number of effective cases)/total number of cases $\times 100 \%$.

\section{Video fluoroscopic swallowing studies (VFSS)}

VFSS were performed using the Shimadzu VS-20D Digital Gastrointestinal X-ray Machine (DR Instrument, Japan). Swallowing function before and after treatment were analyzed. For the oral phase, if patients could not pass food from the mouth into the pharynx, they scored 0 points. If food could not be passed into the pharynx, or the patient could only pass food in the form of a paste into the pharynx, they scored 1 point. If food could be passed into the pharynx but required more than one attempt, the patient scored 2 points. If there was residue food after the swallowing attempt, patients scored 3 points. In the pharyngeal phase, if the deglutition reflex was insufficient, the patient scored 0 points. If a large big amount of residual food was detected in the laryngopharyngeal fossa and piriform fossa, the patients scored 1 point. If a small amount of residual food was detected in the laryngopharyngeal fossa 
Table 1 General clinical data of the patients

\begin{tabular}{lccccc}
\hline Group & $\mathrm{N}$ & Male/female & Age (years) & Illness duration (weeks) & Hemiparesis limb (left/right) \\
\hline TCM & 20 & $11 / 9$ & $63.18 \pm 1.94$ & $3.72 \pm 1.34$ & $12 / 8$ \\
tDCS & 20 & $10 / 10$ & $62.94 \pm 2.08$ & $3.61 \pm 1.27$ & $11 / 9$ \\
Combined & 20 & $12 / 8$ & $64.05 \pm .0 .84$ & $3.58 \pm 1.19$ & $10 / 10$ \\
\hline
\end{tabular}

TCM, traditional Chinese medicine; tDCS, transcranial direct current stimulation; Combined, the TCM treatment combined with tDCS treatment.

and piriform fossa, the patient scored 2 points. If food was passed into the esophagus with one swallowing attempt, the patient scored 3 points. If the patient experienced accidental aspiration, but no choking, they scored 0 points. If the patient experienced accidental aspiration and choking, they scored 1 point. If the patient experienced a low level of aspirations without choking, they scored 2 points. If the patient experienced a small degree of accidental aspiration with choking, they scored 3 points. If there is no accidental aspiration, the patients scored 4 points (8).

\section{Functional magnetic resonance examination}

MRI (1.5T full-body magnetic resonance scanner and head orthogonal coil; GE Healthcare, Chicago, USA ) was performed on patients upon admission and after treatment. MRI scans included pre-contrast axial T1-weighted spinecho imaging (T1WI) with repeat time/echo time (TR/ TE) of $500 \mathrm{~ms} / 14 \mathrm{~ms}$, thickness of $5 \mathrm{~mm}$, no slice gap spacing, and field of view of $240 \mathrm{~mm} \times 240 \mathrm{~mm}$. Functional images were acquired with a gradient echo and echo planar sequence at intervals of 3 seconds (TR/TE, $3,000 / 30 \mathrm{msec}$; slice thickness no slice gap; flip angle $90^{\circ}$; matrix $64 \times 64$ ). In this study, blood oxygenation level-dependent functional images were acquired throughout the experiment and preprocessing images acquired were converted to analyze format using MRIcro software. The neural functional pathways were analyzed by comparing the brain areas before and after activation.

\section{Statistical analysis}

All parameters at each voxel were performed by deconvolution to extract mean activation maps superimposed on the standardized three-dimensional anatomical map. Brain regions were reported on the basis of coordinates using the Talairach Daemon client (7). All data were analyzed using the SPSS18.0 software. The measurement data were expressed as mean \pm standard deviation $(\bar{x} \pm s)$, and comparisons were performed using an independent sample $t$-test. Enumeration data were expressed as rate (\%), and the Chi-square test was used. $\mathrm{P}<0.05$ was considered as statistically significant.

\section{Results}

\section{General clinical data of the patients}

Among the three groups, there were no significant differences in gender, age, illness duration, nor hemiparesis of the limbs $(\mathrm{P}>0.05$; Table 1$)$. The Consolidated Standards of Reporting Trials (CONSORT) diagram is shown in Figure 1.

\section{The results of the WST before and after treatment}

The WST demonstrated that patients given the combined TCM and tDCS treatment had an effective rate of $90.000 \%$, which was significantly higher than the effective rate observed in the TCM group $(65.000 \% ; \mathrm{P}<0.05)$ and the tDCS group $(70.000 \% ; \mathrm{P}<0.05$; Table 2$)$.

\section{The results of VFSS before and after treatment}

The typical performance of VFSS was shown in Figure 2. The VFSS results demonstrated that there were no significance differences in the oral phase, pharyngeal phase, nor the degree of accidental aspiration before treatment among the three groups $(\mathrm{P}>0.05)$. However, after treatment the VFSS score in the combined treatment group was significantly higher than those in the TCM group and the tDCS groups $(\mathrm{P}<0.05$; Table 3$)$.

\section{The results of MRI before and after treatment}

The MRI results demonstrated that the activate brain areas of patients in the TCM group were mainly distributed in 


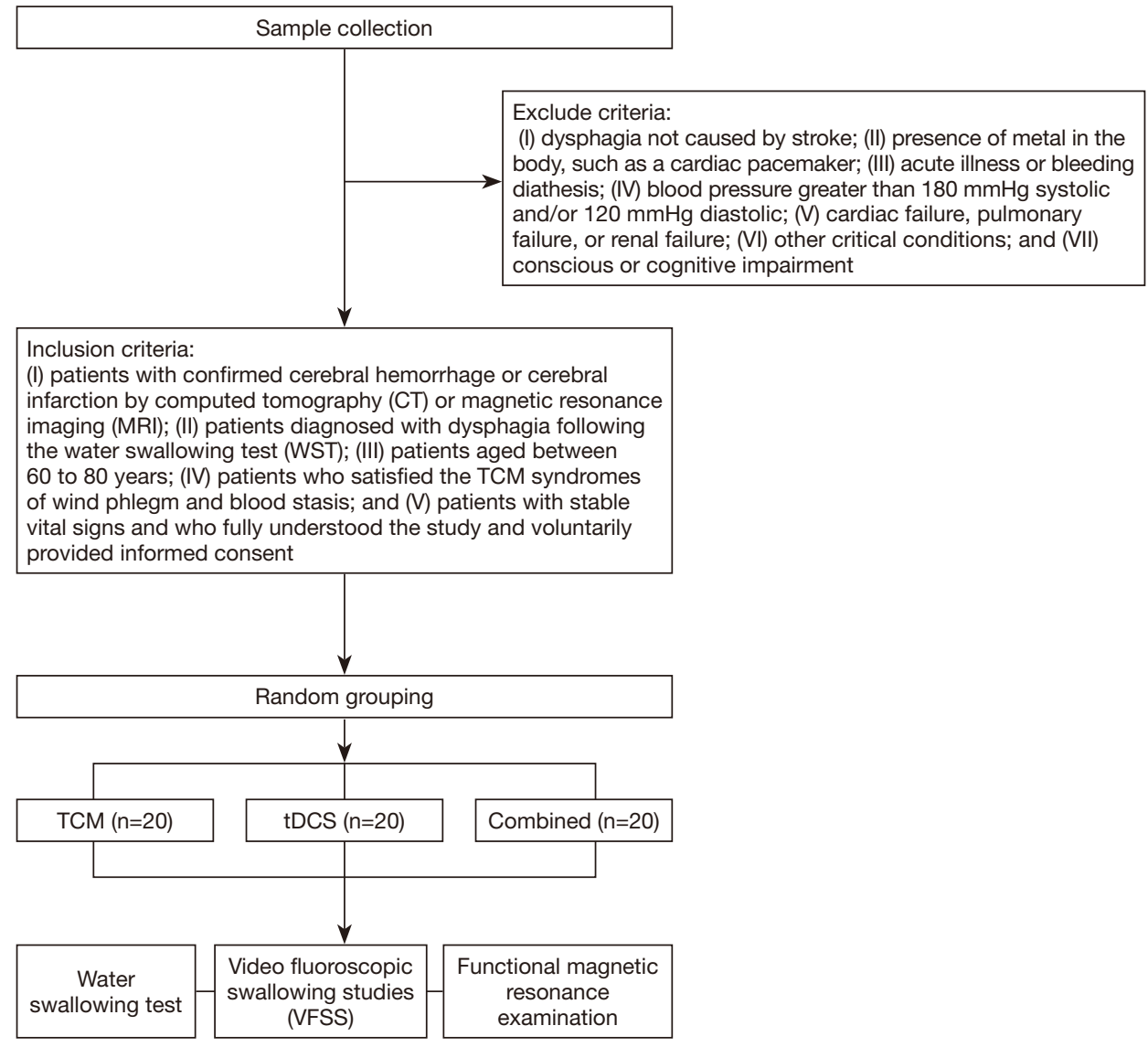

Figure 1 The Consolidated Standards of Reporting Trials (CONSORT) flow diagram of study participants. TCM, traditional Chinese medicine; tDCS, transcranial direct current stimulation.

Table 2 The results of the water swallowing test before and after treatment (N, number of cases)

\begin{tabular}{lcccccc}
\hline Group & N & Cure & Obvious effect & Effective & Ineffective & Effective rate (\%) \\
\hline TCM & 20 & 2 & 5 & 6 & 7 & $65.00^{\mathrm{a}}$ \\
tDCS & 20 & 3 & 4 & 7 & 6 & $70.00^{\mathrm{a}}$ \\
Combined & 20 & 8 & 6 & 4 & 2 & $90.00^{\mathrm{ab}}$ \\
\hline
\end{tabular}

${ }^{\mathrm{a}}, \mathrm{P}<0.05$, comparison in the same group before and after treatment; ${ }^{\mathrm{b}}, \mathrm{P}<0.05$, comparison of the Combined group with the TCM and tDCS groups after treatment. TCM, traditional Chinese medicine; tDCS, transcranial direct current stimulation; Combined, the TCM treatment combined with $\mathrm{tDCS}$ treatment.

the motor area of the left and right precentral gyrus, and the nodules of the left cerebellum posterior lobe. The activate brain areas of patients in the tDCS group were mainly distributed in the left and right precentral gyrus, adjacent bilateral anterior operculum insulae, and left internal frontal gyrus. The activate brain areas of patients in the combined treatment group were mainly distributed in the left and right precentral gyrus, adjacent bilateral anterior operculum insulae, the left and right motor area, the nodules of the left and right cerebellum posterior lobe, the inferior semi-lunar lobule of the left and right cerebellum posterior lobe, and the right midbrain. Therefore, the volume of activate brain area in patients in the combined treatment group was significantly greater than those of patients in the TCM group and the tDCS group $(\mathrm{P}<0.05$; Table 4). 


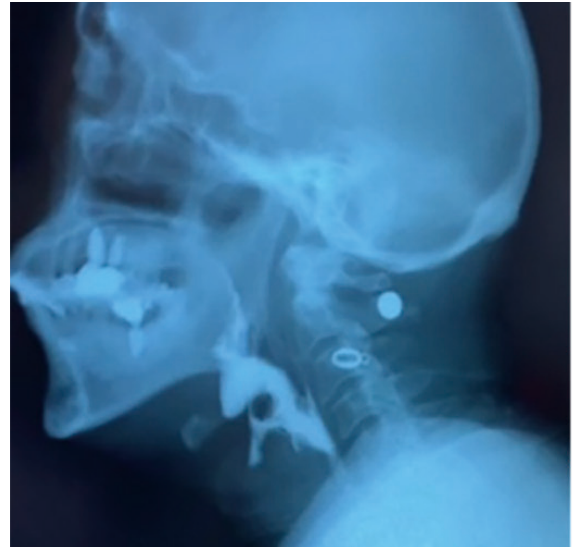

Leakage

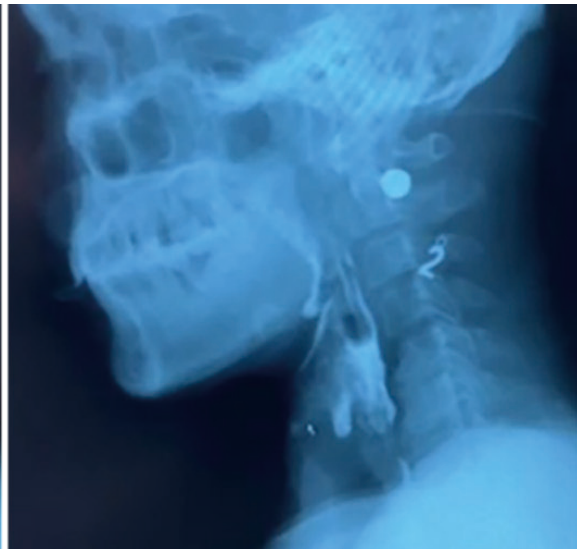

Aspiration

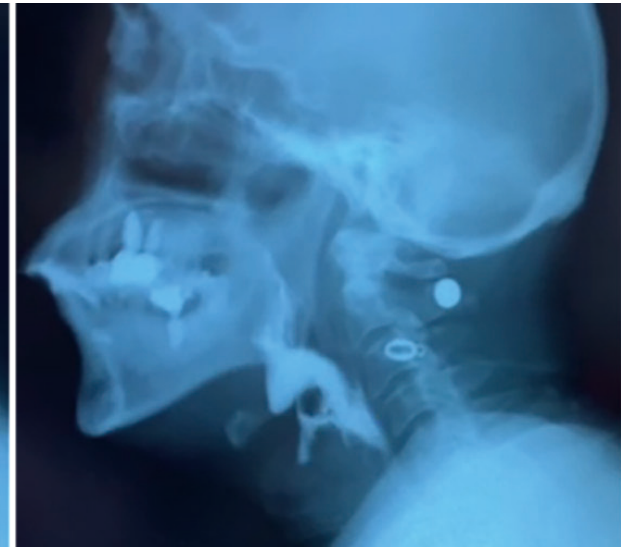

The cricopharyngeal muscle is not open at all

Figure 2 The typical performance of VFSS. VFSS, video fluoroscopic swallowing studies.

Table 3 The results of VFSS before and after treatment

\begin{tabular}{|c|c|c|c|c|c|c|c|}
\hline Group & Counts & \multicolumn{2}{|c|}{ Oral phase } & \multicolumn{2}{|c|}{ Pharyngeal phase } & \multicolumn{2}{|c|}{ Degree of accidental aspiration } \\
\hline TCM & 20 & $0.87 \pm 0.18$ & $1.54 \pm 0.50^{\mathrm{a}}$ & $0.73 \pm 0.21$ & $1.41 \pm 0.27^{\mathrm{a}}$ & $1.09 \pm 0.38$ & $1.81 \pm 0.59^{\mathrm{a}}$ \\
\hline tDCS & 20 & $1.02 \pm 0.20$ & $1.79 \pm 0.49^{a}$ & $0.88 \pm 0.10$ & $1.88 \pm 0.43^{a}$ & $0.99 \pm 0.24$ & $2.04 \pm 0.63^{a}$ \\
\hline Combined & 20 & $0.95 \pm 0.19$ & $2.21 \pm 0.74^{\mathrm{ab}}$ & $0.80 \pm 0.19$ & $2.61 \pm 0.82^{\mathrm{ab}}$ & $1.05 \pm 0.29$ & $2.94 \pm 0.95^{\mathrm{ab}}$ \\
\hline
\end{tabular}

${ }^{a}, \mathrm{P}<0.05$, comparison in the same group before and after treatment; ${ }^{\mathrm{b}}, \mathrm{P}<0.05$, comparison of the Combined group with the TCM group and the tDCS group after treatment. VFSS, video fluoroscopic swallowing studies; TCM, traditional Chinese medicine; tDCS, transcranial direct current stimulation; Combined, the TCM treatment combined with tDCS treatment.

\section{Discussion}

Dysphagia after a stroke is a common poor prognostic factor, induced by limited reflex activity and dysfunction of the swallowing muscles after cerebrovascular damage. It can directly lead to malnutrition, aspiration pneumonia, obstruction, and suffocation (9). Currently, local cold stimulations and tDCS are suitable diagnostic tools for dysphagia in severe stroke patients (10-12).

Transcranial direct current stimulation (tDCS) is an emerging non-invasive brain stimulation technology. It can directly act on the cerebral cortex through a weak current, adjust the excitability of the cerebral cortex, activate the cortical center directionally, and promote the remodeling of the injured center (6). Compared with traditional physical therapy methods such as transcranial magnetic stimulation and deep brain stimulation, the subjects' headache, dizziness, nausea, and insomnia were greatly reduced $(7,13)$.

Sensory stimulation of traditional Chinese medicine can enhance the sensitivity of the soft palate and pharynx, promote the occurrence of swallowing reflex, and can make the brainstem and cerebral cortex perceive stimulation, improve swallowing and eating attention, so as to accelerate the reconstruction of swallowing pathways and improve swallowing function.

This current study demonstrated that patients in the combined group had superior WST and VFSS scores, and greater active brain volume compared to patients in the TCM group and tDCS group $(\mathrm{P}<0.05)$. There may be several reasons for this. Cold stimulation with the Jieyudan powder stick results in contraction of the oral muscles. The sensitivity of the muscles and mucous membranes are effectively increased, thereby promoting a speech reflex (14). Furthermore, when concentrated on the open-tongue, the cold Jieyudan stick can elicit an expectorant effect. According to TCM theory, the Jieyudan powder sticks restore the swallowing function through the interaction with the meridian, which are channels through which qi and other substances flow. However, the volume of the 


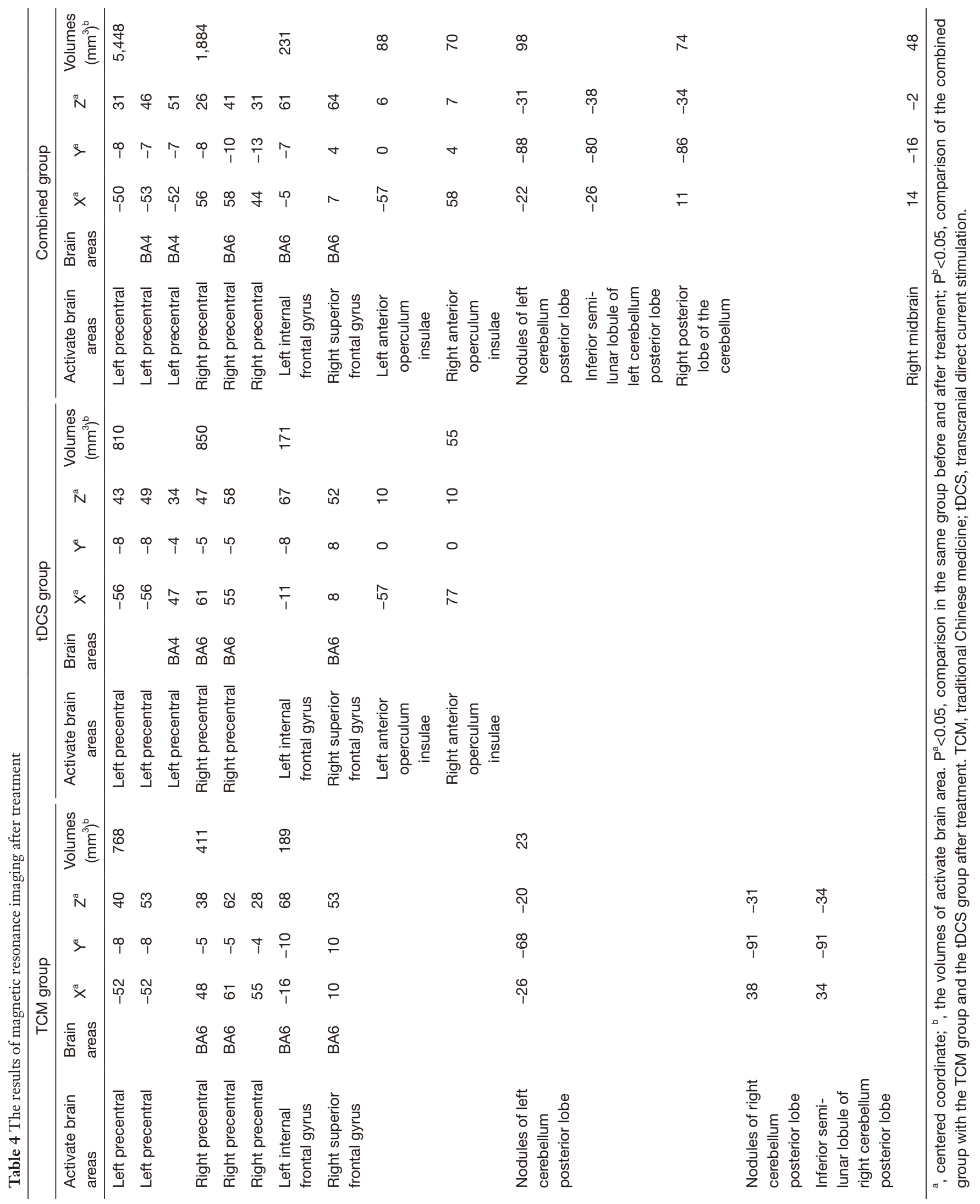


brain activate area was small in the TCM group, which may be due to the fact that the sensory stimulation of TCM mainly acted on the local muscle mucosa, with no obvious effects on the reflex mechanisms of the brain, and little effect on dendritic lateral branch germination and synaptic threshold.

tDCS is a noninvasive rehabilitation approach. Anodic stimulation can cause depolarization of resting membrane potential and improve cortical excitability. Interestingly, contralateral motor-evoked potential amplitude can be induced in the period of transcorpus callosum inhibition and initial latency, which can relieve dysphagia (15-17). However, anodic stimulation was not as effective as cold stimulation. The combined treatment group showed the common WST and VFSS scores, the brain activation volume of both the TCM group and the tDCS group. The sensory stimulation of TCM enhanced pharyngeal sensitivity, and at the same time the dominant areas in the cerebral cortex responsible for the swallowing muscles were activated by transcranial direct current stimulation.

In summary, TCM sensory stimulation combined with tDCS can be utilized to improve deglutition and related complications in stroke patients with dysphagia, and its clinical application should be encouraged.

\section{Acknowledgments}

Funding: This work was supported by the 2021 Zhejiang Traditional Chinese Medicine Scientific Research Fund Project (2021ZB146) and the Zhejiang Provincial Natural Science Foundation of China (No. LQ19H170002).

\section{Footnote}

Reporting Checklist: The authors have completed the CONSORT reporting checklist. Available at http://dx.doi. org/10.21037/apm-21-1055

Trial Protocol: Available at http://dx.doi.org/10.21037/apm21-1055

Data Sharing Statement: Available at http://dx.doi. org/10.21037/apm-21-1055

Conflicts of Interest: All authors have completed the ICMJE uniform disclosure form (available at http://dx.doi. org/10.21037/apm-21-1055). The authors have no conflicts of interest to declare.
Ethical Statement: The authors are accountable for all aspects of the work in ensuring that questions related to the accuracy or integrity of any part of the work are appropriately investigated and resolved. All procedures performed in this study involving human participants were in accordance with the Declaration of Helsinki (as revised in 2013). The study was approved by the Research Ethics Committee of the Third Affiliated Hospital of Zhejiang Chinese Medical University (No.: ZSLL-JS-2020-002-01) and informed consent was taken from all the patients.

Open Access Statement: This is an Open Access article distributed in accordance with the Creative Commons Attribution-NonCommercial-NoDerivs 4.0 International License (CC BY-NC-ND 4.0), which permits the noncommercial replication and distribution of the article with the strict proviso that no changes or edits are made and the original work is properly cited (including links to both the formal publication through the relevant DOI and the license). See: https://creativecommons.org/licenses/by-nc-nd/4.0/.

\section{References}

1. Schöberl F, Ringleb PA, Wakili R, et al. Juvenile Stroke. Dtsch Arztebl Int 2017;114:527-34.

2. Katan M, Luft A. Global Burden of Stroke. Semin Neurol 2018;38:208-11.

3. Hankey GJ. Stroke. Lancet 2017;389:641-54.

4. Chen L, Fang J, Ma R, et al. Additional effects of acupuncture on early comprehensive rehabilitation in patients with mild to moderate acute ischemic stroke: a multicenter randomized controlled trial. BMC Complement Altern Med 2016;16:226.

5. Liu T, Ding Y, Wen A. Traditional Chinese medicine for ischaemic stroke. Lancet Neurol 2018;17:745.

6. Hummel F, Celnik P, Giraux P, et al. Effects of noninvasive cortical stimulation on skilled motor function in chronic stroke. Brain 2005;128:490-9.

7. Tadini L, El-Nazer R, Brunoni AR, et al. Cognitive, mood, and electroencephalographic effects of noninvasive cortical stimulation with weak electrical currents. J ECT 2011;27:134-40.

8. Talairach J, Toumoux P. Co-planar stereotaxic atlas of the human brain. New York: Thieme Medical Publishers, 1988.

9. Cohen DL, Roffe C, Beavan J, et al. Post-stroke dysphagia: A review and design considerations for future trials. Int $\mathrm{J}$ Stroke 2016;11:399-411. 
10. Wang Z, Song WQ, Wang L. Application of noninvasive brain stimulation for post-stroke dysphagia rehabilitation. Kaohsiung J Med Sci 2017;33:55-61.

11. Kwon SH, Seo HG. Unusual Cause of Dysphagia in a Post-Stroke Patient. Dysphagia 2017;32:721-3.

12. Geiger M, Supiot A, Zory R, et al. The effect of transcranial direct current stimulation ( $\mathrm{tDCS}$ ) on locomotion and balance in patients with chronic stroke: study protocol for a randomised controlled trial. Trials 2017;18:492.

13. Mahoney JJ 3rd, Hanlon CA, Marshalek PJ, et al. Transcranial magnetic stimulation, deep brain stimulation, and other forms of neuromodulation for substance use disorders: Review of modalities and implications for treatment. J Neurol Sci 2020;418:117149.

14. Peng W, Lauche R, Ferguson C, et al. Efficacy of Chinese herbal medicine for stroke modifiable risk factors: a

Cite this article as: Lu Y, Zhou W, Lin Y, Du Y, Zhang $X$. The effects of traditional Chinese medicine sensory stimulation combined with transcranial direct current stimulation on deglutition and related complications in stroke patients with dysphagia: a randomized trialx. Ann Palliat Med 2021;10(6):6597-6605. doi: 10.21037/apm-21-1055 systematic review. Chin Med 2017;12:25.

15. Elsner B, Kwakkel G, Kugler J, et al. Transcranial direct current stimulation (tDCS) for improving capacity in activities and arm function after stroke: a network metaanalysis of randomised controlled trials. J Neuroeng Rehabil 2017;14:95.

16. Elsner B, Kugler J, Pohl M, et al. Transcranial direct current stimulation (tDCS) for improving aphasia in adults with aphasia after stroke. Cochrane Database Syst Rev 2019;5:CD009760.

17. Kang N, Summers JJ, Cauraugh JH. Transcranial direct current stimulation facilitates motor learning post-stroke: a systematic review and meta-analysis. J Neurol Neurosurg Psychiatry 2016;87:345-55.

(English Language Editor: J. Teoh) 